\title{
DEVELOPMENT OF LOCAL, GLOBAL, AND TRACE ESTIMATES FOR THE SOLUTIONS OF ELLIPTIC EQUATIONS
}

\author{
MOULAY D. TIDRIRI
}

Received 21 January 2001

\section{Introduction}

In this paper, we improve and extend the local, global, and trace estimates for the solutions of elliptic equations we developed in [6]. These estimates are developed for the solutions of elliptic second-order equations, with source terms, with Dirichlet-Neumann boundary conditions and Dirichlet boundary conditions. They are obtained under quasi-optimal regularity conditions on the source terms. The previous trace estimates we developed in [6] are based on our improved version of the estimates of Aleksandrov, Bakelman, and Pucci estimates $[1,2]$. They are improvement in the sense that they are independent of the low, "relaxation," term of the equations. These later estimates are important in many applications, in particular, for the treatment of quasilinear and fully nonlinear equations. The trace estimates we developed are themselves of great importance in elliptic theory. They are also important in many other applications, in particular, for the study of fixed point methods, the treatment of contraction properties for some operators, and also for the analysis of algorithms in numerical analysis. We have already given some applications of the trace estimates of [6] in [4].

In Section 2, we state a local estimate for elliptic equations. In Section 3, we introduce the first and second basic problems corresponding, respectively, to elliptic equations with Dirichlet-Neumann and Dirichlet-Dirichlet boundary conditions. In Section 4, we state our trace estimates for the first and second basic problems. In Section 5, we state and prove various local and global estimates for the first basic problem. In Section 6, we prove the trace estimate for the first basic problem. In Section 7, we state and prove various local and global estimates for the second basic problem. In Section 8, we prove the trace estimate for the second basic problem. In Section 9, we introduce the third basic problem corresponding to elliptic equations with Dirichlet-Neumann boundary 
132 Local, global, and trace estimates

conditions. In Section 10, we give the trace estimates for the third basic problem. In Section 11, we state and prove various local and global estimates for the third basic problem. In Section 12, we give indications on the proof of the trace estimates for the third basic problem.

\section{A local estimate}

In this section, we present a local estimate that we have developed in [6] for an arbitrary elliptic operator of second order satisfying some conditions to be precised below.

Let $L$ be an operator of the form

$$
L u=a^{i j}(x) D_{i j} u+b^{i}(x) D_{i} u+c(x) u
$$

for any $u$ in $W^{2, n}(\Omega)$, with $\Omega$ a bounded domain of $\mathbb{R}^{n}$. The coefficients $a^{i j}, b^{i}$, and $c, i, j=1, \ldots, n$, are defined on $\Omega$. As usual, the repeated indices indicate a summation from 1 to $n$.

We suppose that the operator $L$ is strictly elliptic in $\Omega$ in the sense that the matrix $\mathscr{A}$ of coefficients $\left[a^{i j}\right]$ is strictly positive everywhere in $\Omega$. Let $\lambda$ and $\Lambda$ denote the smallest and the largest eigenvalue of $\mathscr{A}$, respectively. Let $\mathscr{D}$ denote the determinant of the matrix $\mathscr{A}$ and $\mathscr{D}^{*}=\mathscr{D}^{1 / n}$. We have

$$
0<\lambda \leq \mathscr{D}^{*} \leq \Lambda
$$

We suppose, in addition, that the coefficients $a^{i j}, b^{i}$, and $c$ are bounded in $\Omega$, and that there exist two positive real numbers $\gamma$ and $\delta$ such that

$$
\begin{gathered}
\frac{\Lambda}{\lambda} \leq \gamma, \quad(L \text { is uniformly elliptic }) \\
\left(\frac{|b|}{\lambda}\right)^{2} \leq \delta
\end{gathered}
$$

Our local estimate is stated in the following theorem.

THEOREM 2.1. Let $u \in W^{2, n}(\Omega)$ and suppose that $L u \geq f$ with $f \in L^{n}(\Omega)$ and $c \leq 0$. Then for all spheres $B=B_{2 R}(y)$ of center $y$ and radius $2 R$ included in $\Omega$ and for all $p>0$, we have

$$
\sup _{B_{R}(y)} u \leq C_{R}\left\{\left(\frac{1}{|B|} \int_{B}\left(u^{+}\right)^{p}\right)^{1 / p}+\frac{R}{\lambda}\|f\|_{L^{n}(B)}\right\},
$$

where the constant $C_{R}$ depends on $\left(n, \gamma, \delta R^{2}, p\right)$, but is independent of $c$, and $u^{+}=\max (u, 0)$. 


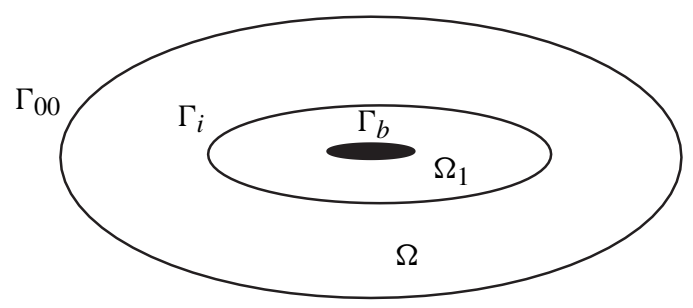

Figure 2.1. Description of the domain $\Omega$ and its splitting.

Remark 2.2. The statement of the same theorem can be found in [3], under the assumption

$$
\frac{|c|}{\lambda} \leq \delta^{\prime}
$$

However, in [3], the constant $C_{R}$ depends indirectly on $c$ through $\delta^{\prime}$. Our estimate is independent on the low relaxation term $c$. This is very important for the applications of these estimates to various problems.

Estimates of this type have many important applications to quasilinear elliptic equations (and systems) and nonlinear elliptic equations (and systems). They have also important applications to the problem of boundary regularity. We will see in the next sections some applications of these estimates to the development of new trace estimates for elliptic equations of second order. The new trace estimates of the next sections are themselves important in elliptic theory. They have important applications in proving contraction properties for some operator and in the analysis of algorithms in numerical analysis. We refer to [5, 6] for further details.

\section{First and second basic problems}

Let $\Omega$ and $\Omega_{l}$ be two connected domains of $\mathbb{R}^{n}$ with $\Omega_{l} \subset \Omega$ (Figure 2.1). Let $\Gamma_{b}, \Gamma_{i}$, and $\Gamma_{\infty}$ denote the boundaries of the two domains

$$
\begin{array}{ll}
\Gamma_{b}=\partial \Omega \cap \partial \Omega_{l} & \text { (internal boundary), } \\
\Gamma_{i}=\partial \Omega_{l} \cap \Omega & \text { (interface), } \\
\Gamma_{\infty}=\partial \Omega \backslash \Gamma_{b} & \text { (farfield boundary). }
\end{array}
$$

Let $n$ denote the external unit normal vector to $\partial \Omega$ or $\partial \Omega_{l}$. Let $V \in\left(L^{\infty}(\Omega)\right)^{n}$ be a given velocity field of an inviscid incompressible flow such that

$$
\operatorname{div} V=0 \quad \text { in } \Omega, \quad V \cdot n=0 \quad \text { on } \Gamma_{b} .
$$


134 Local, global, and trace estimates

The first basic problem is a Dirichlet-Neumann problem,

$$
\begin{gathered}
\mathscr{L} v=-v \Delta v+V \cdot \nabla v+c v=f \quad \text { in } \Omega, \\
v=0 \quad \text { on } \Gamma_{\infty}, \quad \frac{\partial v}{\partial n}=g \quad \text { on } \Gamma_{b},
\end{gathered}
$$

where $g$ is given in $H^{-1 / 2}\left(\Gamma_{b}\right)$, the coefficient $c$ is strictly positive, and $v$ is the diffusion coefficient. We assume that $f \in L^{n}(\Omega) \cap L^{\infty}(\Omega)$. Let $W$ be the subspace of $H^{1}(\Omega)$ defined by

$$
W=\left\{w \in H^{1}(\Omega) \mid w=0 \text { on } \Gamma_{\infty}\right\} .
$$

We define two bilinear forms on $W$

$$
a(v, w)=\int_{\Omega} v \nabla v \nabla w+\int_{\Omega} \operatorname{div}(V v) w, \quad(v, w)=\int_{\Omega} v w .
$$

The weak formulation of the first basic problem (3.3) and (3.4) is to find $v \in W$ satisfying

$$
a(v, w)+c(v, w)=\int_{\Gamma_{b}} v g w d \Gamma+\int_{\Omega} f w, \quad \forall w \in W .
$$

We write $c$ in the form $c=1 / \tau$ where $\tau$ is positive and we assume that the coefficients $v$ and $\tau$ satisfy

$$
\nu \tau \leq \frac{1}{2}, \quad \tau \leq 1
$$

This hypothesis is not necessary but simplifies the proofs to come. Moreover, it is not restrictive (see [4, 6]).

The second basic problem is a Dirichlet-Dirichlet problem,

$$
\begin{gathered}
\mathscr{L} v=-v \Delta v+V \cdot \nabla v+c v=f \quad \text { in } \Omega_{l}, \\
v=h \quad \text { on } \Gamma_{i}, \quad v=0 \quad \text { on } \Gamma_{b},
\end{gathered}
$$

where $h$ is given in $H^{1 / 2}\left(\Gamma_{i}\right)$, the coefficient $c$ is strictly positive, and $v$ is the diffusion coefficient. We assume that $f \in L^{n}\left(\Omega_{l}\right) \cap L^{\infty}\left(\Omega_{l}\right)$ and the velocity field $V$ is given by (3.2). We write the coefficient $c$ in the form $c=1 / \tau$ and we assume that the coefficients $v$ and $\tau$ satisfy (3.8). Let $W$ be the subspace of $H^{1}\left(\Omega_{l}\right)$ defined by

$$
W=\left\{w \in H^{1}\left(\Omega_{l}\right) \mid w=0 \text { on } \Gamma_{b}\right\} .
$$

We introduce two bilinear forms on $W$

$$
a_{l}(v, w)=v \int_{\Omega_{l}} \nabla v \cdot \nabla w+\int_{\Omega_{l}} \operatorname{div}(V v) w, \quad(v, w)=\int_{\Omega_{l}} v w .
$$




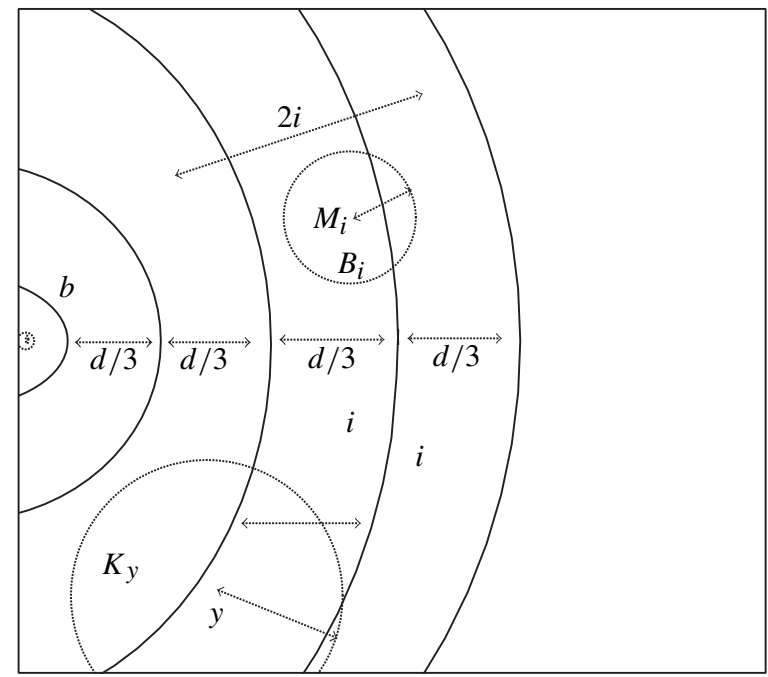

Figure 4.1. Description of the domain $\Omega_{l}$ and the splitting used in the majorization of the local solution.

The weak formulation of the second basic problem (3.9) and (3.10) is to find $v \in W$ such that

$$
\begin{gathered}
a_{l}(v, w)+\left(\frac{1}{\tau}\right)(v, w)=\int_{\Gamma_{i}} v \frac{\partial v}{\partial n} w+\int_{\Omega_{l}} f_{l} w, \quad \forall w \in W, \\
\left.v\right|_{\Gamma_{i}}=h,
\end{gathered}
$$

where $h$ is given in $H^{1 / 2}\left(\Gamma_{i}\right)$.

For the elliptic operators (3.3) and (3.9), the coefficients introduced in Section 2 can be expressed explicitly. We have $L=-\mathscr{L}$. Therefore, $\lambda=\Lambda=v$ and we choose $\gamma=1, \delta=n\left(\|V\|_{\infty} / \nu\right)^{2}$.

\section{Trace estimates I}

We present in this section our trace estimates for the solutions of the first and second basic problems. We first introduce some geometric notations. These are necessary for the statement of the trace estimates and their proofs that will be given in the next sections. Let $d$ denote the distance between $\Gamma_{b}$ and $\Gamma_{i}$. Let $\Omega_{i}$ be the subdomain of $\Omega_{l}$ of width $d / 3$ with external boundary $\Gamma_{i}$ (Figure 4.1). Let $y \in \Omega_{i}$ and $K_{y}=B_{d / 3}(y)$ be the sphere of center $y$ and radius $d / 3$. There exist $y_{1}, \ldots, y_{l} \in \Omega_{i}$ such that

$$
\Omega_{2 i}=\cup_{y \in \Omega_{i}} B_{d / 6}(y) \subset \cup_{j=1}^{l} K_{y_{j}} .
$$

We then define $K$ by setting

$$
K=\cup_{j=1}^{l} K_{y_{j}} .
$$


Assume that $d$ satisfies $2 d<\operatorname{dist}\left(\Gamma_{b}, \Gamma_{\infty}\right)$. This assumption ensures that $B_{2 d / 3}(y) \subset \Omega$ for any point $y \in \Omega_{i}$. It is not necessary since we can modify the radius of the sphere $K_{y}=B_{q d / 3}(y)$ such that $B_{2 q d / 3}(y) \subset \Omega$. However, it simplifies the notations in the proof.

Let $\Gamma_{V}$ be the center surface of $\Omega_{l}$ defined as the surface whose distance from $\Gamma_{b}$ and $\Gamma_{i}$ is at least $d / 2$. Let $\Omega_{i l}$ be the subdomain of $\Omega_{l}$ of width $d / 6$ centered at $\Gamma_{V}$. Let $y \in \Gamma_{V}$ and let $K_{l y}=B_{d / 4}(y)$ be the sphere of radius $d / 4$. We then introduce $K_{l}$ which is constructed in the same way as the set $K$ above. We also introduce a set $\Omega_{b}$ which is a subdomain of $\Omega_{l}$ whose internal boundary is $\Gamma_{b}$ and such that its external boundary is of constant distance from $\Gamma_{b}$ with a distance of $d / 4$.

Let $\beta$ be a real number such that

$$
0<\beta<\frac{3 \sqrt{v}}{d},
$$

and set

$$
k=\frac{\beta}{v \sqrt{\tau}} .
$$

We now state the trace estimates.

THEOREM 4.1. The solution $v$ of the first basic problem (3.7) satisfies

$$
\begin{aligned}
\|v\|_{1 / 2, \Gamma_{i}} \leq & C_{1} \sqrt{d}\left(d+\frac{\|V\|_{\infty}}{v}\right)^{1 / 2} \exp \left(-\frac{k d^{2}}{36}\right) \\
& \times\left[\|g\|_{-1 / 2, \Gamma_{b}}+\frac{1}{v}\|f\|_{0, \Omega}+\frac{d}{v}\|f\|_{L^{n}(\Omega)}+2 \tau\|f\|_{\infty, \Omega}\right]+\frac{C_{2}}{\sqrt{v}}\|f\|_{0},
\end{aligned}
$$

where $C_{1}$ and $C_{2}$ are constants, with $C_{1}$ depending only on $n$ and $\left(\|V\|_{\infty} d / v\right)^{2}$, but not on $\tau$.

THEOREM 4.2. The solution $v$ of the second basic problem (3.13) and (3.14) satisfies

$$
\begin{aligned}
\left\|\frac{\partial v}{\partial n}\right\|_{-1 / 2, \Gamma_{b}} \leq & C_{1} \alpha_{1}^{2} \alpha_{2} \exp \left(-\frac{k d^{2}}{36}\right)\|h\|_{1 / 2, \Gamma_{i}} \\
& +\alpha_{1}\left(C_{1} \alpha_{2} \exp \left(-\frac{k d^{2}}{36}\right) \frac{1}{v}+C_{2} \frac{1}{\sqrt{v}}\right)\left\|f_{l}\right\|_{0, \Omega_{l}} \\
& +C_{1} \alpha_{1} \alpha_{2} \exp \left(-\frac{k d^{2}}{36}\right) \frac{d}{v}\left\|f_{l}\right\|_{L^{n}\left(\Omega_{l}\right)} \\
& +C_{1} \alpha_{1} \alpha_{2} \exp \left(-\frac{k d^{2}}{36}\right) \tau\left\|f_{l}\right\|_{\infty, \Omega_{i}},
\end{aligned}
$$


where $C_{1}$ and $C_{2}$ are constants with $C_{1}$ depending only on $n$ and $\left(n\|V\|_{\infty} d / v\right)^{2}$, $\alpha_{1}=\left[1+(1 / v)\|V\|_{\infty, \Omega_{l}}+1 / \nu \tau\right]$ and $\alpha_{2}=\sqrt{d}\left(d+\|V\|_{\infty} / v\right)^{1 / 2}$.

In these estimates we observe that the dependence in terms of the low relaxation terms $(1 / \tau)$ is completely explicit. The practical applications of these estimates can be for example the study of approximations in time methods when the time step (that can be taken here to be $\tau$ ) goes to 0 . They can also be applied to the study of the convergence properties of operators $[4,5]$. Moreover, they can be used for other purposes such as proving existence results. We refer to $[4,5]$ for more details.

\section{Local and global estimates for the first basic problem}

The first basic result states a global $H^{1}$ estimate of the solution $v$ of the first basic problem (3.7) in terms of the boundary data $g$ and the data $f$.

LEMMA 5.1. There exists a constant $c_{0}$ such that

$$
\|v\|_{1, \Omega} \leq c_{0}\|g\|_{-1 / 2, \Gamma_{b}}+\frac{1}{v}\|f\|_{0}
$$

Proof. Proceeding as in the proof of Lemma 3.1 of [6], we obtain

$$
\int_{\Omega}\left\{v|\nabla v|^{2}+\left(\frac{1}{\tau}\right) v^{2}\right\}=\int_{\Gamma_{b}} v g v+\int_{\Omega} f v .
$$

Using Cauchy-Schwarz inequality, the trace theorem, and (3.8) we obtain

$$
\begin{aligned}
\|v\|_{1, \Omega}^{2} & \leq\|g\|_{-1 / 2, \Gamma_{b}}\|v\|_{1 / 2, \Gamma_{b}}+\frac{1}{v}\|f\|_{0}\|v\|_{0} \\
& \leq C(\Omega)\|g\|_{-1 / 2, \Gamma_{b}}\|v\|_{1, \Omega}+\frac{1}{v}\|f\|_{0}\|v\|_{0} .
\end{aligned}
$$

Hence we have

$$
\|v\|_{1, \Omega} \leq c_{0}\|g\|_{-1 / 2, \Gamma_{b}}+\frac{1}{v}\|f\|_{0}
$$

The next lemma states a local estimate of the solution $v$ of the first basic problem (3.7).

Lemma 5.2. There exists a constant $c_{1}$ such that

$$
\|v\|_{\infty, K} \leq c_{1}\|v\|_{0, \Omega}+c_{1} \frac{d}{v}\|f\|_{L^{n}(\Omega)},
$$

where $c_{1}$ depends only on $n$ and $\left(\|V\|_{\infty} d / v\right)^{2}$. 
Proof. The operator

$$
L=-\mathscr{L}
$$

satisfies the assumptions of Theorem 2.1 with $c=-1 / \tau$. Applying this theorem with $p=2, y \in \Omega_{i}$, and $K_{y}=B_{d / 3}(y)\left(B_{2 d / 3}(y) \subset \Omega\right.$ since $\left.2 d<\operatorname{dist}\left(\Gamma_{b}, \Gamma_{\infty}\right)\right)$, we obtain

$$
\|v\|_{\infty, K y} \leq c_{1}\|v\|_{0, B_{2 d / 3}(y)}+c_{1} \frac{d}{v}\|f\|_{L^{n}(\Omega)} .
$$

Therefore we obtain

$$
\|v\|_{\infty, K y} \leq c_{1}\|v\|_{0, \Omega}+c_{1} \frac{d}{v}\|f\|_{L^{n}(\Omega)}
$$

where $c_{1}$ is a constant depending only on $n$ and $\left(\|V\|_{\infty} d / v\right)^{2}$.

Applying (5.8) to each $K_{y_{j}}$ we obtain

$$
\|v\|_{\infty, K} \leq \sup _{j=1, \ldots, l} c_{1 j}\left(\|v\|_{0, \Omega}+\frac{d}{v}\|f\|_{L^{n}(\Omega)}\right) .
$$

Setting $c_{1}=\sup _{j=1, \ldots, l} c_{1 j}$, we finally obtain

$$
\|v\|_{\infty, K} \leq c_{1}\|v\|_{0, \Omega}+c_{1} \frac{d}{v}\|f\|_{L^{n}(\Omega)} .
$$

And the lemma is proved.

We now establish other local estimates for the solution $v$ of the first basic problem. For any $M_{i}$ in $\Omega_{i}$, we introduce (see Figure 4.1) a ball $B_{i}$ centered at $M_{i}$ of radius $d / 6$ and $v_{i}=\exp \left[k\left(r^{2}-d^{2} / 36\right)\right]\left(\|v\|_{\infty, \partial B_{i}}+2 \tau\|f\|_{\infty, B_{i}}\right)$.

We have the following result.

\section{LEMMA 5.3. The solution $v$ of the first basic problem satisfies}

$$
\|v\|_{\infty, \Omega_{i}} \leq \exp \left(-\frac{k d^{2}}{36}\right)\left(\|v\|_{\infty, \partial B_{i}}+2 \tau\|f\|_{\infty, B_{i}}\right) .
$$

Proof. To prove this lemma we use the same ideas we introduced to derive Lemma 3.3 in [6]. For the convenience of the reader we give detailed proof.

The operator $\mathscr{L}$ applied to $v_{i}$ can be written in polar coordinates (with $r=$ $\left.M_{i} M\right)$

$$
\mathscr{L} v_{i}=4\left(-k^{2} v r^{2}-k v+\frac{k}{2} V \cdot e_{r} r+\frac{1}{4 \tau}\right) v_{i}
$$

Therefore

$$
\mathscr{L} v_{i} \geq 4\left(-k^{2} v r^{2}-\frac{k}{2}\left|V \cdot e_{r}\right| r+\left(\frac{1}{4 \tau}-k v\right)\right) v_{i} .
$$


We introduce the function

$$
\varphi(r, k)=a(k) r^{2}+b(k) r+c(k),
$$

with

$$
a(k)=-k^{2} v, \quad b(k)=-\frac{k}{2}\left|V \cdot e_{r}\right|, \quad c(k)=\frac{1}{8 \tau}-k v .
$$

We seek to satisfy the following relation:

$$
0 \leq \inf \varphi(r, k) \text { for } 0 \leq r \leq \frac{d}{6} .
$$

As $\varphi(r, k)$ decreases on $\mathbb{R}^{+}$, this will be satisfied if and only if

$$
\varphi\left(\frac{d}{6}\right) \geq 0,
$$

that is, if and only if

$$
-\frac{k^{2} v d^{2}}{36}-\frac{k d\|V\|}{12}+\frac{1}{8 \tau}-k v \geq 0 .
$$

Replacing $k$ by its value, this becomes

$$
-\frac{\beta^{2} d^{2}}{(36 v \tau)}-\frac{\beta d\|V\|}{12 v \sqrt{\tau}}+\frac{1}{8 \tau}-\frac{\beta v}{v \sqrt{\tau}} \geq 0 .
$$

Multiplying by $\sqrt{\tau}$, it follows that

$$
\frac{1}{4 \sqrt{\tau}}\left(\frac{1}{2}-\frac{\beta^{2} d^{2}}{9 v}\right) \geq \beta\left(1+\frac{d\|V\|}{12 v}\right) .
$$

The constraint $\beta<3 \sqrt{v} / d$ finally yields after division

$$
\varphi(r, k) \geq 0 \quad \text { iff } \frac{1}{4 \sqrt{\tau}} \geq \beta\left[1+\frac{d\|V\|}{12 v}\right]\left[\frac{1}{2}-\frac{\beta^{2} d^{2}}{9 v}\right]^{-1} .
$$

From the relation (5.13), we deduce that for $\beta<3 \sqrt{v} / d$ and $\tau$ satisfying (5.21), we have

$$
L v_{i} \geq \frac{1}{2 \tau} v_{i} \geq L v .
$$

In addition, by construction,

$$
v_{i} \geq v \quad \text { on } \partial B_{i}
$$

Consequently, using the maximum principle we obtain

$$
v \leq v_{i} \quad \text { in } B_{i} .
$$


140 Local, global, and trace estimates

In particular

$$
v\left(M_{i}\right) \leq \exp \left(-\frac{k d^{2}}{36}\right)\left[\|v\|_{\infty, \partial B_{i}}+2 \tau\|f\|_{\infty, B_{i}}\right]
$$

Repeating the same process for $-v$, we finally obtain

$$
\left|v\left(M_{i}\right)\right| \leq \exp \left(-\frac{k d^{2}}{36}\right)\left[\|v\|_{\infty, \partial B_{i}}+2 \tau\|f\|_{\infty, B_{i}}\right], \quad \forall M_{i} \in \Omega_{i} .
$$

We then obtain the estimate (5.11).

We now give an $H^{1}$ estimate of the solution $v$ of the first basic problem on the domain $\Omega_{\infty}=\Omega \backslash \Omega_{l}$.

Lemma 5.4. There exist constants $c_{2}$ and $c_{3}$ such that

$$
\|v\|_{1, \Omega_{\infty}} \leq c_{2}\|v\|_{\infty, \Omega_{i}} \sqrt{d}\left(d+\frac{\|V\|_{\infty}}{v}\right)^{1 / 2}+\frac{c_{3}}{\sqrt{v}}\|f\|_{0} .
$$

Proof. Let $\xi \in H^{1}(\Omega)$ be such that

$$
\xi=1 \text { in } \Omega_{\infty}, \quad \operatorname{supp} \xi \subset \Omega_{i} \cup \Omega_{\infty}
$$

Proceeding as in the proof of Lemma 3.4 in [6], we obtain

$$
\begin{aligned}
\int_{\Omega} f \xi^{2} v= & \int_{\Omega_{\infty}} v\left(|\nabla v|^{2}+|v|^{2}\right)+\int_{\Omega_{i}} v\left(|\nabla(\xi v)|^{2}+|\xi v|^{2}\right) \\
& +\int_{\Omega}\left(\frac{1}{\tau}-v\right) \xi^{2} v^{2}-\int_{\Omega_{i}}\left(v v^{2}|\nabla \xi|^{2}+v^{2} \xi V \cdot \nabla \xi\right) .
\end{aligned}
$$

Hence, we obtain

$$
\begin{aligned}
& v\|v\|_{1, \Omega_{\infty}}^{2}+\int_{\Omega_{i}} v\left(|\nabla(\xi v)|^{2}+|\xi v|^{2}\right)+\int_{\Omega}\left(\frac{1}{\tau}-v\right) \xi^{2} v^{2} \\
& \leq \int_{\Omega_{i}}\left(v v^{2}|\nabla \xi|^{2}+v^{2} \xi V \cdot \nabla \xi\right)+\frac{1}{2} \int_{\Omega} \xi^{2} f^{2}+\frac{1}{2} \int_{\Omega} \xi^{2} v^{2} .
\end{aligned}
$$

The relation (3.8) then yields

$$
v\|\xi v\|_{1, \Omega_{\infty} \cup \Omega_{i}}^{2} \leq\|v\|_{\infty, \Omega_{i}}^{2}|\xi|_{1, \Omega_{i}}^{2}\left(v+\frac{\|\xi\|_{0, \Omega_{i}}\|V\|_{\infty}}{|\xi|_{1, \Omega_{i}}}\right)+\frac{1}{2} \int_{\Omega} \xi^{2} f^{2} .
$$

If we take $\xi$ bounded such that

$$
\|\xi\|_{0, \Omega_{i}} \leq 1, \quad|\xi|_{1, \Omega_{i}}=c_{2} d
$$


where $c_{2}$ is a constant, (5.31) then becomes

$$
\|v\|_{1, \Omega_{\infty}} \leq c_{2}\|v\|_{\infty, \Omega_{i}} \sqrt{d}\left(d+\frac{\|V\|_{\infty}}{v}\right)^{1 / 2}+\frac{c_{3}}{\sqrt{v}}\|f\|_{0}
$$

and this concludes the proof.

\section{Proof of Theorem 4.1}

In this section we prove Theorem 4.1. Since $\partial B_{i} \subset K$, we have

$$
\|v\|_{\infty, \partial B_{i}} \leq\|v\|_{\infty, K} .
$$

Lemma 5.2 then yields

$$
\|v\|_{\infty, \partial B_{i}} \leq c_{1}\|v\|_{0, \Omega}+c_{1} \frac{d}{v}\|f\|_{L^{n}(\Omega)}
$$

Using Lemma 5.3 we obtain

$$
\|v\|_{\infty, \Omega_{i}} \leq \exp \left(-\frac{k d^{2}}{36}\right)\left[\|v\|_{\infty, \partial B_{i}}+2 \tau\|f\|_{\infty, B_{i}}\right] .
$$

Combining the last two estimates, we obtain

$$
\|v\|_{\infty, \Omega_{i}} \leq c_{1} \exp \left(-\frac{k d^{2}}{36}\right)\left[\|v\|_{0, \Omega}+\frac{d}{v}\|f\|_{L^{n}(\Omega)}+2 \tau\|f\|_{\infty, \Omega_{i}}\right] .
$$

Applying Lemma 5.1 we get

$\|v\|_{\infty, \Omega_{i}} \leq c_{1} \exp \left(-\frac{k d^{2}}{36}\right)\left[c_{0}\|g\|_{-1 / 2, \Gamma_{b}}+\frac{1}{v}\|f\|_{0}+\frac{d}{v}\|f\|_{L^{n}(\Omega)}+2 \tau\|f\|_{\infty, \Omega_{i}}\right]$.

An application of Lemma 5.4 yields

$$
\begin{aligned}
\|v\|_{1, \Omega_{\infty}} \leq & c_{2} \sqrt{d}\left(d+\frac{\|V\|_{\infty}}{v}\right)^{1 / 2} c_{1} \exp \left(-\frac{k d^{2}}{36}\right) \\
& \times\left[c_{0}\|g\|_{-1 / 2, \Gamma_{b}}+\frac{1}{v}\|f\|_{0}+\frac{d}{v}\|f\|_{L^{n}(\Omega)}+2 \tau\|f\|_{\infty, \Omega_{i}}\right]+c_{3} \frac{1}{\sqrt{v}}\|f\|_{0} .
\end{aligned}
$$

To conclude we use the trace theorem which yields $\|v\|_{1 / 2, \Gamma_{i}} \leq c_{4}\|v\|_{1, \Omega_{\infty}}$

$$
\begin{aligned}
\leq & c_{0} c_{1} c_{4} c_{2} \sqrt{d}\left(d+\frac{\|V\|_{\infty}}{v}\right)^{1 / 2} \exp \left(-\frac{k d^{2}}{36}\right) \\
& \times\left[\|g\|_{-1 / 2, \Gamma_{b}}+\frac{1}{v}\|f\|_{0, \Omega}+\frac{d}{v}\|f\|_{L^{n}(\Omega)}+2 \tau\|f\|_{\infty, \Omega}\right]+\frac{c_{3} c_{4}}{\sqrt{v}}\|f\|_{0}
\end{aligned}
$$

which corresponds to our theorem with $C_{1}=c_{0} c_{1} c_{4} c_{2}$ and $C_{2}=c_{3} c_{4}$. 
142 Local, global, and trace estimates

We can obtain the explicit dependence of $C_{1}$ in terms of $n$ and $\left(n\|V\|_{\infty} d / v\right)^{2}$. Since in our Lemmas 5.1, 5.2, 5.3, and 5.4 the constants are explicit, all we need is to make explicit the dependence of $C_{R}$ in terms of $\left(n, \gamma, \delta R^{2}, p\right)$ in Theorem 2.1 of [6]. This is possible by looking more closely at the proof of this theorem [6]. However, our main goal here is to derive a trace estimate in which the dependence in terms of $\tau$ only is explicit. We observe that the trace estimate and the other local and global estimates are obtained under no restrictions on $\tau$ or any other parameters (except those appearing in the statement of the first and second basic problems).

In the case where $f \equiv 0$ we obtain estimates which are improved versions of the estimates we have developed in [6]. For $f \neq 0$, the estimates we obtain here represent an extension of our previous estimates [6] to elliptic equations with source terms.

\section{Local and global estimates for the second basic problem}

We first state a global estimate for the solution $v$ of the second basic problem (3.13) and (3.14).

LEMMA 7.1. The solution $v$ of the second basic problem (3.13) and (3.14) satisfies

$$
\|v\|_{1, \Omega_{l}} \leq\left[1+\frac{c_{1}}{v}\|V\|_{\infty, \Omega_{l}}+\frac{1}{v \tau}\right]\|h\|_{1 / 2, \Gamma_{i}}+\frac{c_{1}}{v}\|f\|_{0, \Omega_{l}} .
$$

Proof. Choosing $w=v$ in (3.13) we obtain

$$
\int_{\Omega_{l}}|\nabla v|^{2}+\frac{1}{v \tau} \int_{\Omega_{l}} v^{2}=\int_{\Gamma_{i}} \frac{\partial v}{\partial n} h+\frac{1}{v} \int_{\Omega_{l}} f v-\frac{1}{v} \int_{\Omega_{l}} v \operatorname{div}(V v) .
$$

Using Cauchy-Schwarz inequality, (3.2), and (3.10), we obtain

$$
\begin{aligned}
\int_{\Omega_{l}}|\nabla v|^{2}+\frac{1}{v \tau} \int_{\Omega_{l}} v^{2} \\
\quad \leq\left\|\frac{\partial v}{\partial n}\right\|_{-1 / 2, \Gamma_{i}}\|h\|_{1 / 2, \Gamma_{i}}+\frac{1}{v}\|f\|_{0, \Omega_{l}}\|v\|_{0, \Omega_{l}}+\frac{1}{2 v}\left|\int_{\Gamma_{i}} V \cdot n h^{2}\right| \\
\quad \leq\left\|\frac{\partial v}{\partial n}\right\|_{-1 / 2, \Gamma_{i}}\|h\|_{1 / 2, \Gamma_{i}}+\frac{1}{v}\|f\|_{0, \Omega_{l}}\|v\|_{0, \Omega_{l}}+\frac{1}{2 v}\|V \cdot n\|_{\infty, \Gamma_{i}}\|h\|_{1 / 2, \Gamma_{i}}^{2} .
\end{aligned}
$$

The term $\|\partial v / \partial n\|_{-1 / 2, \Gamma_{i}}$ is estimated as follows. Using (3.2), (3.10), and (3.13), we obtain

$$
\int_{\Gamma_{i}} \frac{\partial v}{\partial n} w=\int_{\Omega_{l}} \nabla v \nabla w+\frac{1}{v} \int_{\Omega_{l}} w V \cdot \nabla v-\frac{1}{v} \int_{\Omega_{l}} f w+\frac{1}{v \tau} \int_{\Omega_{l}} v w .
$$


Using the trace theorem and (3.2), we obtain

$$
\left|\int_{\Gamma_{i}} \frac{\partial v}{\partial n} w\right| \leq\left(\left(1+\frac{1}{v}\|V\|_{\infty, \Omega_{l}}\right)\|\nabla v\|_{0, \Omega_{l}}+\frac{1}{v}\|f\|_{0, \Omega_{l}}+\frac{1}{v \tau}\|v\|_{0, \Omega_{l}}\right)\|w\|_{1, \Omega_{l}} .
$$

Therefore, we have

$$
\left\|\frac{\partial v}{\partial n}\right\|_{-1 / 2, \Gamma_{i}} \leq\left(1+\frac{1}{v}\|V\|_{\infty, \Omega_{l}}\right)\|\nabla v\|_{0, \Omega_{l}}+\frac{1}{v \tau}\|v\|_{0, \Omega_{l}}+\frac{1}{v}\|f\|_{0, \Omega_{l}} .
$$

Combining (7.3) and (7.6), and using (3.8) and the trace theorem, we obtain

$$
\begin{aligned}
\|v\|_{1, \Omega_{l}}^{2} \leq & \left\|\frac{\partial v}{\partial n}\right\|_{-1 / 2, \Gamma_{i}}\|h\|_{1 / 2, \Gamma_{i}} \\
& +\frac{1}{v}\|f\|_{0, \Omega_{l}}\|v\|_{0, \Omega_{l}}+\frac{1}{2 v}\|V \cdot n\|_{\infty, \Gamma_{i}}\|h\|_{1 / 2, \Gamma_{i}}^{2} \\
\leq & {\left[\left(1+\frac{1}{v}\|V\|_{\infty, \Omega_{l}}+\frac{1}{v \tau}\right)\|h\|_{1 / 2, \Gamma_{i}}\right.} \\
& \left.+\frac{c_{1}}{v}\|V\|_{\infty, \Omega_{l}}\|h\|_{1 / 2, \Gamma_{i}}+\frac{c_{1}}{v}\|f\|_{0, \Omega_{l}}\right]\|v\|_{1, \Omega_{l}} .
\end{aligned}
$$

We finally obtain

$$
\|v\|_{1, \Omega_{l}} \leq\left[1+\frac{c_{1}}{v}\|V\|_{\infty, \Omega_{l}}+\frac{1}{v \tau}\right]\|h\|_{1 / 2, \Gamma_{i}}+\frac{c_{1}}{v}\|f\|_{0, \Omega_{l}}
$$

which is the required estimate.

Remark 7.2. Lemma 7.1 represents a major improvement over Lemmas 4.1 and 4.2 of [6] which required the following condition on the diffusion coefficient $v$, the velocity field $V$ and the coefficient $\tau: 1 / \tau \geq v / 2+(1 / 2 v)\|V\|_{\infty}^{2}$.

Let $K_{l y}=B_{d / 4}(y)$ be the sphere centered at $y$ and of radius $d / 4$, with $y$ belonging to $\Gamma_{V}$ (see Figure 7.1). By construction, $\Gamma_{V}$ is the center surface of $\Omega_{l}$, and $\Omega_{i l}$ is the subdomain of width $d / 6$ centered at $\Gamma_{V}$. We have the following lemma.

Lemma 7.3. There exists a constant $c_{2}$ such that

$$
\|v\|_{\infty, K_{l}} \leq c_{2}\|v\|_{0, \Omega_{l}}+c_{2} \frac{d}{v}\|f\|_{L^{n}\left(\Omega_{l}\right)}
$$

where $c_{2}$ depends only on $n$ and $\left(\|V\|_{\infty} d / v\right)^{2}$. 
144 Local, global, and trace estimates

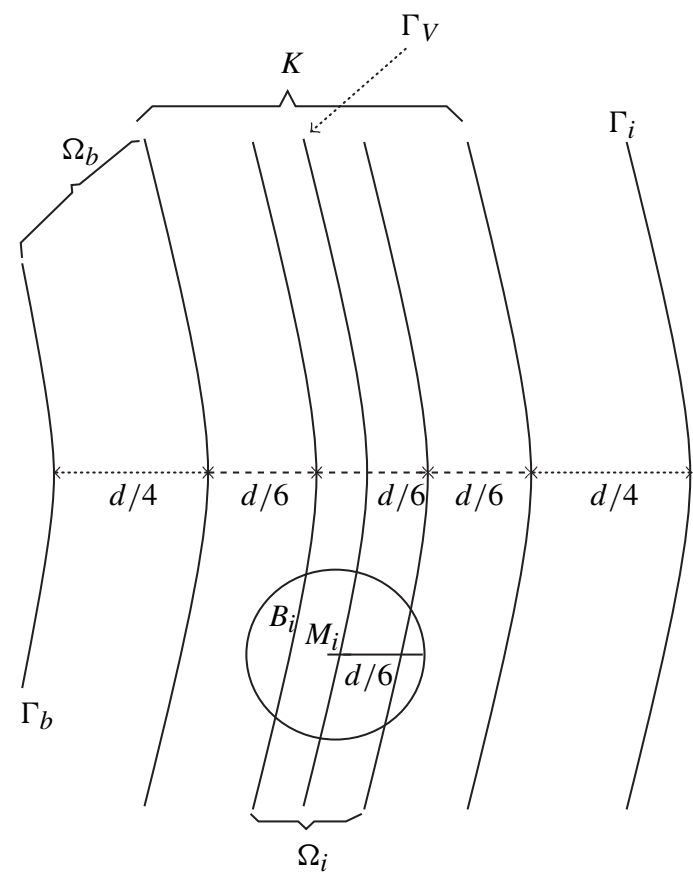

Figure 7.1. Description of the local domain $\Omega_{l}$ and the splitting used in the majorization of the global solution.

Proof of Lemma 7.3. See the proof of Lemma 5.2 in Section 5.

Next we establish another local estimate for the solution of the second basic problem (3.13) and (3.14). For any $M_{i} \in \Omega_{i l}$, we introduce (see Figure 7.1) a ball $B_{i}$ centered at $M_{i}$ and of radius $d / 6$ and the function $v_{i}=\exp \left[k\left(r^{2}-\right.\right.$ $\left.\left.d^{2} / 36\right)\right]\left[\|v\|_{\infty, \partial B_{i}}+2 \tau\|f\|_{\infty, B_{i}}\right]$.

We then have the following result.

LEMMA 7.4. The solution $v$ of the second basic problem (3.13) and (3.14) satisfies

$$
\|v\|_{\infty, \Omega_{i l}} \leq \exp \left[-\frac{k d^{2}}{36}\right]\left[\|v\|_{\infty, \partial B_{i}}+2 \tau\|f\|_{\infty, B_{i}}\right]
$$

Proof of Lemma 7.4. See the proof of Lemma 5.3 in Section 5.

Let $\Omega_{b}$ be the subdomain of $\Omega_{l}$ introduced in Section 4 and described in Figure 7.1. The next result states an $H^{1}$ global estimate of the solution of the second basic problem. 
LEMMA 7.5. The solution $v$ of the second basic problem (3.13) and (3.14) satisfies

$$
\|v\|_{1, \Omega_{b} \cup \Omega_{i l}} \leq c_{3}\|v\|_{\infty, \Omega_{i l}} \sqrt{d}\left(d+\frac{\|V\|_{\infty}}{v}\right)^{1 / 2}+\frac{c_{3}}{\sqrt{v}}\|f\|_{0, \Omega_{l}} .
$$

Proof of Lemma 7.5. See the proof of Lemma 5.4 in Section 5.

\section{Proof of Theorem 4.2}

In this section we prove Theorem 4.2. Since $\partial B_{i} \subset K_{l}$ by construction, Lemmas 7.3 and 7.4 imply

$$
\|v\|_{\infty, \Omega_{i l}} \leq c_{2} \exp \left(-\frac{k d^{2}}{36}\right)\left[\|v\|_{0, \Omega_{l}}+\frac{d}{v}\|f\|_{L^{n}\left(\Omega_{l}\right)}+2 \tau\|f\|_{\infty, B_{i}}\right] .
$$

Furthermore, Lemma 7.1 yields

$$
\begin{aligned}
\|v\|_{\infty, \Omega_{i l}} \leq c_{2} \exp \left(-\frac{k d^{2}}{36}\right) & \left(\left[1+\frac{c_{1}}{v}\|V\|_{\infty, \Omega_{l}}+\frac{1}{v \tau}\right]\|h\|_{1 / 2, \Gamma_{i}}\right. \\
& \left.+\frac{c_{1}}{v}\|f\|_{0, \Omega_{l}}+\frac{d}{v}\|f\|_{L^{n}\left(\Omega_{l}\right)}+2 \tau\|f\|_{\infty, \Omega_{i l}}\right) .
\end{aligned}
$$

Using Lemma 7.5 we obtain

$$
\begin{aligned}
\|v\|_{1, \Omega_{b} \cup \Omega_{i l} \leq} \leq & c_{3}\|v\|_{\infty, \Omega_{i l}} \sqrt{d}\left(d+\frac{\|V\|_{\infty}}{v}\right)^{1 / 2}+\frac{c_{3}}{\sqrt{v}}\|f\|_{0, \Omega_{l}} \\
\leq & c_{2} c_{3} \sqrt{d}\left(d+\frac{\|V\|_{\infty}}{v}\right)^{1 / 2} \exp \left(-\frac{k d^{2}}{36}\right) \\
& \times\left(\left[1+\frac{c_{1}}{v}\|V\|_{\infty, \Omega_{l}}+\frac{1}{v \tau}\right]\|h\|_{1 / 2, \Gamma_{i}}\right. \\
& \left.+\frac{c_{1}}{v}\|f\|_{0, \Omega_{l}}+\frac{d}{v}\|f\|_{L^{n}\left(\Omega_{l}\right)}+2 \tau\|f\|_{\infty, \Omega_{i}}\right) \\
& +\frac{c_{3}}{\sqrt{v}}\|f\|_{0, \Omega_{l}} .
\end{aligned}
$$

Before concluding we establish an estimate of the term

$$
\left\|\frac{\partial v}{\partial n}\right\|_{-1 / 2, \Gamma_{b}} .
$$

Choosing $w$ such that

$$
w \in H^{1}\left(\Omega_{b}\right) \text { with } w=0 \text { on } \partial \Omega_{b} \backslash \partial \Gamma_{b},
$$


and using (3.13) we obtain

$$
\int_{\Omega_{b}}\left(-v \Delta v+\operatorname{div}(V v)+\frac{v}{\tau}\right) w=\int_{\Omega_{b}} f w .
$$

Applying Green's formula and using (3.2), we obtain

$$
\int_{\Gamma_{b}} \frac{\partial v}{\partial n} w=\int_{\Omega_{b}}\left(\nabla v \nabla w+\left(\frac{1}{v}\right) V \cdot \nabla v w+\frac{1}{v \tau} v w\right)-\frac{1}{v} \int_{\Omega_{b}} f w .
$$

As in the proof of Lemma 7.1, we obtain

$$
\left\|\frac{\partial v}{\partial n}\right\|_{-1 / 2, \Gamma_{b}} \leq\left[1+\frac{1}{v}\|V\|_{\infty, \Omega_{b}}+\frac{1}{v \tau}\right]\|v\|_{1, \Omega_{b}}+\frac{1}{v}\|f\|_{0, \Omega_{b}} .
$$

The completion of the proof of the theorem results from the combination of the relations (8.3) with (8.8). We obtain

$$
\begin{aligned}
\left\|\frac{\partial v}{\partial n}\right\|_{-1 / 2, \Gamma_{b}} \leq & c_{1} c_{2} c_{3} \alpha_{1} \alpha_{2} \exp \left(-\frac{k d^{2}}{36}\right) \\
& \times\left(\alpha_{1}\|h\|_{1 / 2, \Gamma_{i}}+\frac{1}{v}\|f\|_{0, \Omega_{l}}+\frac{d}{v}\|f\|_{L^{n}\left(\Omega_{l}\right)}+2 \tau \|_{\infty, \Omega_{i}}\right) \\
& +c_{3} \alpha_{1} \frac{1}{\sqrt{v}}\|f\|_{0, \Omega_{l}}+\frac{1}{v}\|f\|_{0, \Omega_{b}} \\
\leq & C_{1} \alpha_{1}^{2} \alpha_{2} \exp \left(-\frac{k d^{2}}{36}\right)\|h\|_{1 / 2, \Gamma_{i}} \\
& +\alpha_{1}\left(C_{1} \alpha_{2} \exp \left(-\frac{k d^{2}}{36}\right) \frac{1}{v}+C_{2} \frac{1}{\sqrt{v}}\right)\|f\|_{0, \Omega_{l}} \\
& +C_{1} \alpha_{1} \alpha_{2} \exp \left(-\frac{k d^{2}}{36}\right) \frac{d}{v}\|f\|_{L^{n}\left(\Omega_{l}\right)} \\
& +C_{1} \alpha_{1} \alpha_{2} \exp \left(-\frac{k d^{2}}{36}\right) \tau\|f\|_{\infty, \Omega_{i}} .
\end{aligned}
$$

We then obtain the theorem with $C_{1}=c_{1} c_{2} c_{3}$ and $C_{2}=c_{3}$ constants with $C_{1}$ depending only on $n$ and $\left(\|V\|_{\infty} d / v\right)^{2}, \alpha_{1}=\left[1+(1 / v)\|V\|_{\infty, \Omega_{l}}+1 / \nu \tau\right]$ and $\alpha_{2}=\sqrt{d}\left(d+\|V\|_{\infty} / v\right)^{1 / 2}$.

We notice that this theorem represents a major improvement as compared with Theorem 4.1 of [6] which required the assumption $1 / \tau \geq v / 2+(1 / 2 v)\|V\|_{\infty}^{2}$.

In the case where $f \equiv 0$ we obtain estimates which are improved versions of the estimates we have developed in [6]. For $f \neq 0$, the estimates we obtain here are extensions of our previous estimates [6] to elliptic equations with source terms. 


\section{Third basic problem}

Let $\Omega$ be a connected bounded domain of $\mathbb{R}^{n}$, such that its boundary $\partial \Omega$ is Lipschitzian. Let $\Omega_{g}$ and $\Omega_{l}$ be connected domains of $\mathbb{R}^{n}$ with $\Omega_{g} \cup \Omega_{l}=\Omega$ and $\Omega_{g} \cap \Omega_{l}=\emptyset$. Let $\Gamma_{b}=\partial \Omega \cap \partial \Omega_{l}$, (internal boundary), $\Gamma_{i}=\partial \Omega_{l} \cap \Omega=\partial \Omega_{g} \cap \Omega$, (interface), $\Gamma_{\infty}=\partial \Omega \backslash \Gamma_{b}=\partial \Omega_{g} \cap \partial \Omega$ (farfield boundary). We denote by $n$ the external unit normal vector to $\partial \Omega$ or $\partial \Omega_{l}$. Let $V \in\left(L^{\infty}(\Omega)\right)^{n}$ be a velocity field of an inviscid incompressible flow given by (3.2).

The third basic problem is a Dirichlet-Neumann problem

$$
\begin{array}{clrl}
\mathscr{L} v & =-v \Delta v+V \cdot \nabla v+c v=f & \text { in } \Omega_{g}, \\
v=0 & \text { on } \Gamma_{\infty}, \quad \frac{\partial v}{\partial n}=g & \text { on } \Gamma_{i},
\end{array}
$$

where $g$ is given in $H^{-1 / 2}\left(\Gamma_{i}\right)$, the coefficient $c$ is strictly positive, and $v$ is the diffusion coefficient. We assume that $f \in L^{n}\left(\Omega_{g}\right) \cap L^{\infty}\left(\Omega_{g}\right)$. Let $W$ be the subspace of $H^{1}\left(\Omega_{g}\right)$ defined by

$$
W=\left\{w \in H^{1}\left(\Omega_{g}\right) \mid w=0 \text { on } \Gamma_{\infty}\right\} .
$$

We define two bilinear forms on $W$

$$
a(v, w)=\int_{\Omega_{g}} v \nabla v \nabla w+\int_{\Omega_{g}} \operatorname{div}(V v) w, \quad(v, w)=\int_{\Omega_{g}} v w .
$$

The weak formulation of the third basic problem (9.1) and (9.2) is to find $v \in W$ satisfying

$$
a(v, w)+c(v, w)=\int_{\Gamma_{i}} v g w d \Gamma+\int_{\Omega_{g}} f w, \quad \forall w \in W .
$$

As in the previous sections we write $c$ in the form $c=1 / \tau$ where $\tau$ is positive and we assume that the coefficients $v$ and $\tau$ satisfy (3.8). This hypothesis is not necessary but simplifies the proofs to come. Moreover, it is not restrictive (see $[4,6])$.

For the elliptic operators (9.1), the coefficients introduced in Section 2 can be expressed explicitly. We have $L=-\mathscr{L}$. Therefore $\lambda=\Lambda=v$ and we choose $\gamma=1, \delta=n\left(\|V\|_{\infty} / v\right)^{2}$.

\section{Trace estimates II}

We present in this section our trace estimates for the solutions of the third basic problems. We first introduce some geometric notations. These are necessary for the statement of the trace estimates and their proofs that will be given in the next sections.

Let $d$ denote the distance between $\Gamma_{b}$ and $\Gamma_{i}$. Let $\Omega_{i}$ be the subdomain of $\Omega_{l}$ of width $d / 3$ with external boundary $\Gamma_{i}$ (Figure 4.1). Let $y \in \Omega_{i}$ and 
$K_{y}=B_{d / 3}(y)$ be the sphere of center $y$ and radius $d / 3$. There exist $y_{1}, \ldots, y_{l} \in$ $\Omega_{i}$ such that

$$
\Omega_{2 i}=\cup_{y \in \Omega_{i}} B_{d / 6}(y) \subset \cup_{j=1}^{l} K_{y_{j}} .
$$

We then define $K$ by setting

$$
K=\cup_{j=1}^{l} K_{y_{j}} .
$$

Assume that $d$ satisfies $2 d<\operatorname{dist}\left(\Gamma_{b}, \Gamma_{\infty}\right)$. This assumption ensures that $B_{2 d / 3}(y) \subset \Omega$ for any point $y \in \Omega_{i}$. It is not necessary since we can modify the radius of the sphere $K_{y}=B_{q d / 3}(y)$ such that $B_{2 q d / 3}(y) \subset \Omega$. However, it simplifies the notations in the proof.

Let $\beta$ be a real number such that

$$
0<\beta<\frac{3 \sqrt{v}}{d}
$$

and set

$$
k=\frac{\beta}{v \sqrt{\tau}} .
$$

We now state the trace estimates.

THEOREM 10.1. The solution $v$ of the third basic problem (9.5) satisfies

$$
\begin{aligned}
\|v\|_{1 / 2, \Gamma_{i}} \leq & C_{1} \sqrt{d}\left(d+\frac{\|V\|_{\infty}}{v}\right)^{1 / 2} \exp \left(-\frac{k d^{2}}{36}\right) \\
& \times\left[\|g\|_{-1 / 2, \Gamma_{i}}+\frac{2}{v}\|f\|_{0, \Omega}+\frac{d}{v}\|f\|_{L^{n}(\Omega)}+2 \tau\|f\|_{\infty, \Omega}\right]+\frac{C_{2}}{\sqrt{v}}\|f\|_{0},
\end{aligned}
$$

where $C_{1}$ and $C_{2}$ are constants, with $C_{1}$ depending only on $n$ and $\left(\|V\|_{\infty} d / v\right)^{2}$, but not on $\tau$.

In these estimates we observe that the dependence in terms of the low relaxation terms $(1 / \tau)$ is completely explicit. The practical applications of these estimates can be for example the study of approximations in time methods when the time step (that can be taken here to be $\tau$ ) goes to 0 . They can also be applied to the study of the convergence properties of operators such as those in $[4,5]$. Moreover, they can be used for other purposes such as proving existence results. We refer to $[4,5]$ for more details.

\section{Local and global estimates for the third basic problem}

For the third basic problem we obtain similar local and global estimates as in the case of the first basic problem. The proofs of these estimates are similar to those for the first basic problem. The only exception is the global $H^{1}$ estimate stated in Lemma 11.1 below. 
Lemma 11.1. For $\tau$ small there is

$$
\|v\|_{1, \Omega} \leq c_{0}\|g\|_{-1 / 2, \Gamma_{i}}+\frac{1}{v}\|f\|_{0}
$$

where $c_{0}$ is a constant.

Proof. Proceeding as in the proof of Lemma 3.1 of [6], we obtain

$$
\int_{\Omega}\left\{v|\nabla v|^{2}+V \cdot \nabla v v+\left(\frac{1}{\tau}\right) v^{2}\right\}=\int_{\Gamma_{i}} v g v+\int_{\Omega} f v .
$$

We then obtain

$$
\frac{v}{2} \int_{\Omega}|\nabla v|^{2}+\frac{2 v-\tau\|V\|_{\infty}^{2}}{2 v \tau} \int_{\Omega} v^{2}=\int_{\Gamma_{i}} v g v+\int_{\Omega} f v .
$$

Using Cauchy-Schwarz inequality, the trace theorem, and (3.8) we obtain

$$
\begin{aligned}
\|v\|_{1, \Omega}^{2} & \leq 2\|g\|_{-1 / 2, \Gamma_{i}}\|v\|_{1 / 2, \Gamma_{i}}+\frac{2}{v}\|f\|_{0}\|v\|_{0} \\
& \leq C(\Omega)\|g\|_{-1 / 2, \Gamma_{i}}\|v\|_{1, \Omega}+\frac{2}{v}\|f\|_{0}\|v\|_{0} .
\end{aligned}
$$

Hence we have

$$
\|v\|_{1, \Omega} \leq c_{0}\|g\|_{-1 / 2, \Gamma_{i}}+\frac{2}{v}\|f\|_{0}
$$

The next lemma states a local estimate of the solution $v$ of the third basic problem (3.7).

LEMMA 11.2. There exists a constant $c_{1}$ such that

$$
\|v\|_{\infty, K} \leq c_{1}\|v\|_{0, \Omega}+c_{1} \frac{d}{v}\|f\|_{L^{n}(\Omega)},
$$

where $c_{1}$ depends only on $n$ and $\left(\|V\|_{\infty} d / v\right)^{2}$.

Proof. See the proof of Lemma 5.2.

We also have other local estimates for the solution $v$ of the third basic problem. For any $M_{i}$ in $\Omega_{i}$, we introduce (see Figure 4.1) a ball $B_{i}$ centered at $M_{i}$ of radius $d / 6$ and $v_{i}=\exp \left[k\left(r^{2}-d^{2} / 36\right)\right]\left(\|v\|_{\infty, \partial B_{i}}+2 \tau\|f\|_{\infty, B_{i}}\right)$.

We have the following result.

LEMMA 11.3. The solution $v$ of the first basic problem satisfies

$$
\|v\|_{\infty, \Omega_{i}} \leq \exp \left(-\frac{k d^{2}}{36}\right)\left(\|v\|_{\infty, \partial B_{i}}+2 \tau\|f\|_{\infty, B_{i}}\right) .
$$


Proof. See the proof of Lemma 5.3.

We now give an $H^{1}$ estimate of the solution $v$ of the third basic problem on the domain $\Omega_{\infty}=\Omega \backslash \Omega_{l}$.

LEMMA 11.4. There exist constants $c_{2}$ and $c_{3}$ such that

$$
\|v\|_{1, \Omega_{\infty}} \leq c_{2}\|v\|_{\infty, \Omega_{i}} \sqrt{d}\left(d+\frac{\|V\|_{\infty}}{v}\right)^{1 / 2}+\frac{c_{3}}{\sqrt{v}}\|f\|_{0} .
$$

Proof. See the proof of Lemma 5.4.

\section{Proof of Theorem 10.1}

The proof of Theorem 10.1 can be obtained following the same ideas as in the proof of Theorem 4.1. It relies on the various local and global estimates derived in the last section.

\section{Acknowledgement}

The author is partially supported by the Air Force Office of Scientific Research under contract number Grant F49620-99-1-0197.

\section{References}

[1] A. D. Aleksandrov, Majorants of solutions of linear equations of order two, Vestnik Leningrad. Univ. 21 (1966), no. 1, 5-25 (Russian), [English translation in Amer. Math. Soc. Transl. (2) 68 (1968), 120-143. Zbl 177.36803]. MR 33\#7684.

[2] I. J. Bakel'man, On the theory of quasilinear elliptic equations, Sibirsk. Mat. Ž. 2 (1961), 179-186 (Russian). MR 23\#A3900.

[3] D. Gilbarg and N. S. Trudinger, Elliptic Partial Differential Equations of Second Order, 2nd ed., Fundamental Principles of Mathematical Sciences, vol. 224, Springer-Verlag, Berlin, 1983. MR 86c:35035. Zbl 562.35001.

[4] P. Le Tallec and M. D. Tidriri, Application of maximum principles to the analysis of a coupling time marching algorithm, J. Math. Anal. Appl. 229 (1999), no. 1, 158-169. MR 99k:35035. Zbl 922.35029.

[5] M. D. Tidriri, Local, global, and trace estimates for the solutions of uniformly elliptic equations, in preparation.

[6] Local and global estimates for the solutions of convection-diffusion problems, J. Math. Anal. Appl. 229 (1999), no. 1, 137-157. MR 99k:35034. Zbl 924.35046.

Moulay D. Tidriri: Department of Mathematics, Iowa State University, 400 Carver Hall, Ames, IA 50011-2064, USA

E-mail address: tidriri@iastate.edu 


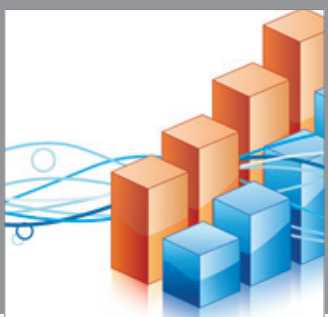

Advances in

Operations Research

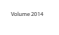

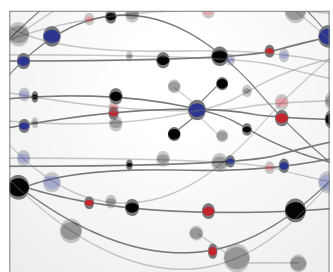

\section{The Scientific} World Journal
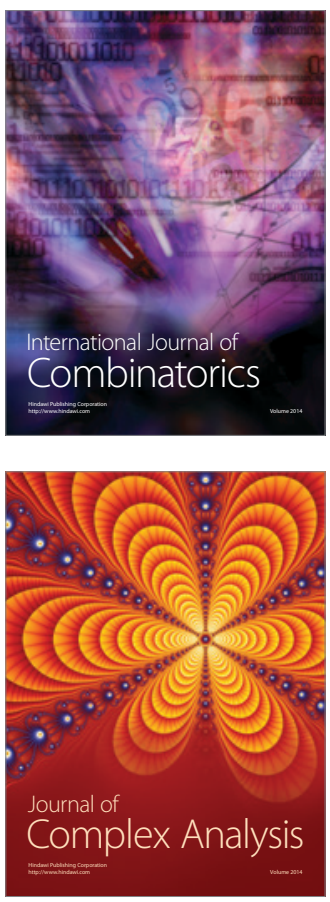

International Journal of

Mathematics and

Mathematical

Sciences
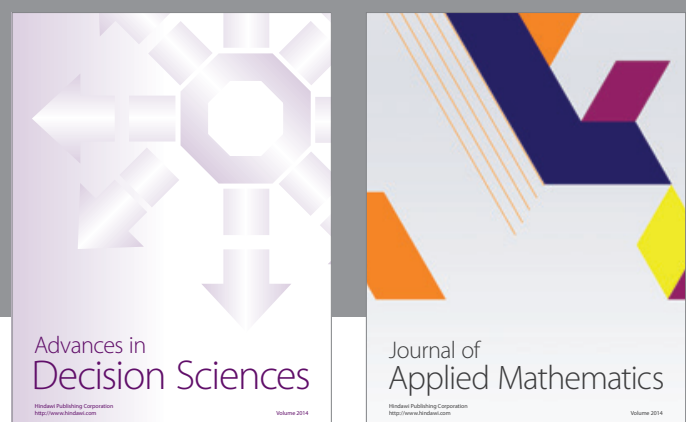

Journal of

Applied Mathematics
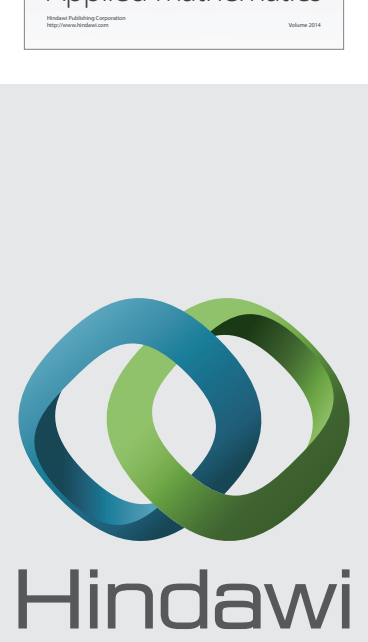

Submit your manuscripts at http://www.hindawi.com
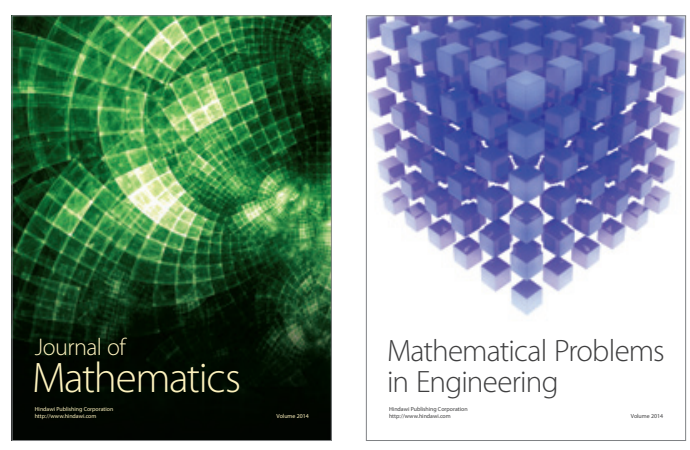

Mathematical Problems in Engineering
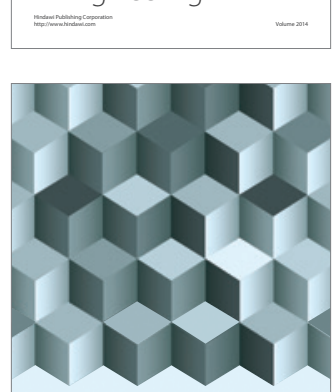

Journal of

Function Spaces
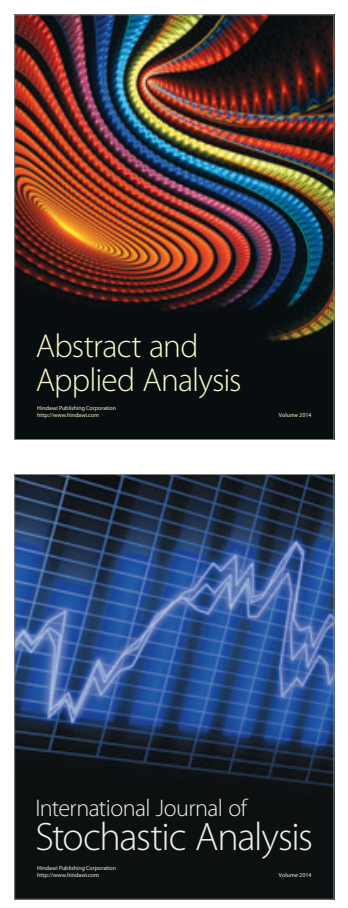

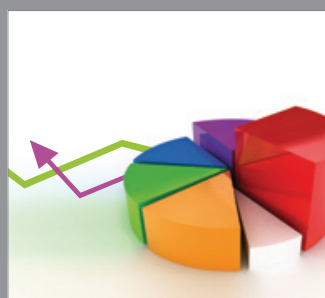

ournal of

Probability and Statistics

Promensencen
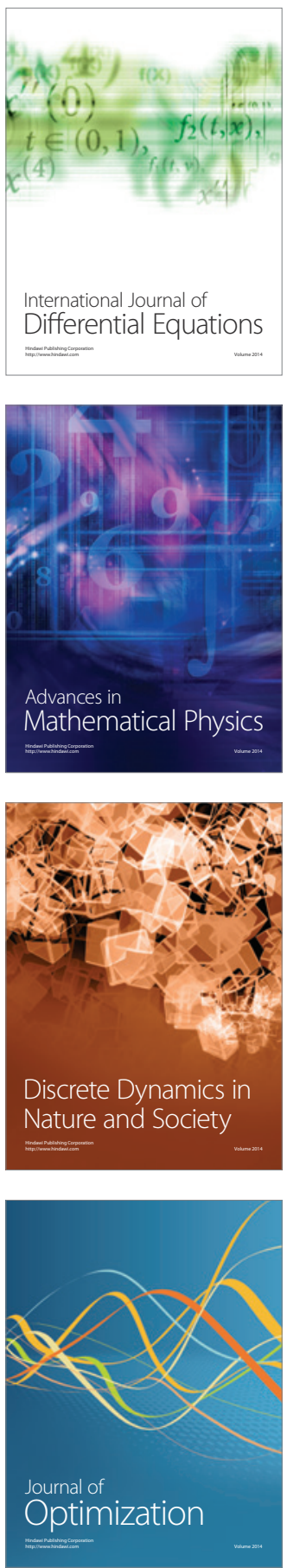